\title{
The Role of Eicosanoids in Regulating the Ubiquitin Proteasome System and Proteostasis
}

\author{
Amanpreet Kaur
}

\begin{abstract}
The ubiquitin proteasome system (UPS) is a protein degradation mechanism in eukaryotes crucial to maintaining protein homeostasis, or proteostasis. There are tissue-specific differences in UPS activity and proteostasis, but the intercellular signalling mechanisms that mediate these differences are not well understood. This work examines eicosanoid signalling molecules-which are derived from polyunsaturated fatty acids (PUFAs) - and their role in proteostasis regulation, particularly the UPS. A reporter transgene that expresses the UbG76V-GFP chimeric protein, a metastable substrate for the UPS, is used in Caenorhabditis elegans epithelial cells to monitor the level of UPS activity. In wild-type nematodes, UbG76V-GFP levels remain high through 24 hours post L4 stage $(L 4+24)$. Then, levels decrease significantly due to increased UPS activity as the animals age and develop 48 hours past L4 (L4+48). Mutants for fat- 1 , a desaturase enzyme that converts $\omega-6$ PUFAs to $\omega-3$ PUFAs, exhibited elevated UbG76V-GFP turnover in the hypodermis even at the L4+24 stage, suggesting that either $\omega-6$ PUFAs (or their eicosanoid derivatives) promote UPS activity or $\omega-3$ PUFAs (or their eicosanoid derivatives) inhibit UPS activity. In the intestine, mutants for fat- 1 showed reduced UbG76V-GFP turnover at the L4+24 and L4+48 life stages. Additionally, mutants for emb-8-an NADPH reductase needed to convert PUFAs into eicosanoids-also showed reduced UbG76V-GFP turnover in the hypodermis even the L4+48 stage. These results suggest that elements of the eicosanoid signalling pathway, including $\omega-6$ PUFAs and their derivatives, significantly contribute to regulation of the UPS and proteostasis.
\end{abstract}

\section{Introduction}

In eukaryotic organisms, protein homeostasis involves the cellular control of protein synthesis, folding, trafficking, and degradation in order to properly control biological processes-specifically, DNA replication, transcription, and mitosis. Rapid cell division during these biological processes can cause unfolded or damaged proteins. The initiation of refolding these proteins, along with the prevention of protein aggregation, is controlled partly by protein degradation mechanisms. Many human diseases are characterized by an inability to degrade damaged or unfolded proteins, particularly those caused by neurodegeneration in the brain. The resultant protein aggregation is often responsible for the neuronal cell death seen in diseases such as Alzheimer's, Parkinson's, and Huntington's, which predominantly affect older populations.

A major mechanism of protein degradation is the ubiquitin proteasome system (UPS). The UPS removes damaged and unfolded target proteins by tagging them with ubiquitin, a small 
protein, resulting in their recognition and digestion by a large proteasome protease complex (Figure 1). In the UPS, an initial ATP hydrolysis step adenylates a molecule of ubiquitin. The ubiquitin is then transferred to the target protein to signal for further polyubiquitination. Once the chain of ubiquitin molecules on the target protein is at least four units long, that substrate becomes a target for degradation by the proteasome. There is a reduction in proteostasis that occurs with age, but the specific changes that occur and the pathways that affect these changes are currently unclear.

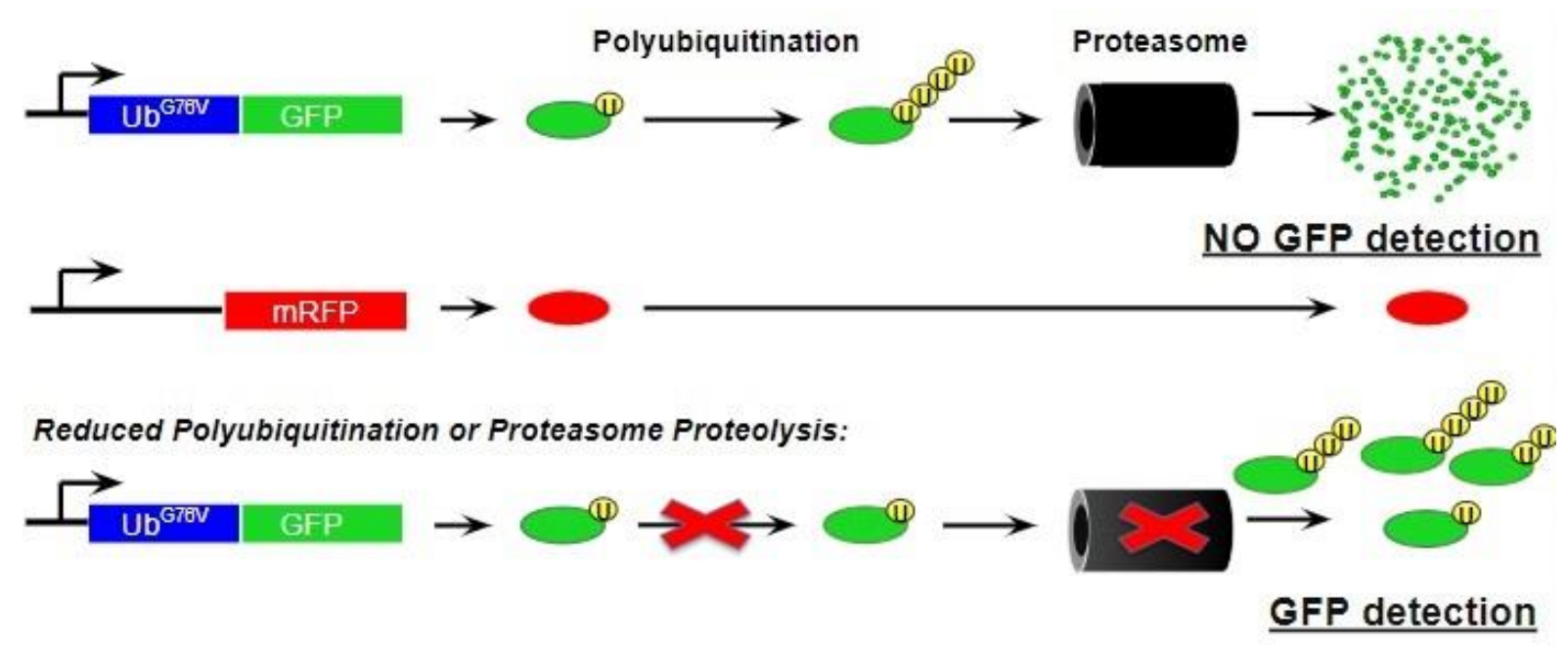

Figure 1. Schematic representation of ubiquitin proteasome system (UPS) activity in the hypodermis. Proper UPS function shows that the unstable UbG76V_GFP (green) transgene is quickly polyubiquitinated and degraded whereas mRFP (red) remains stable. When not functioning properly, UbG76V_GFP is either not polyubiquitinated or not degraded and thus remains stable. Adapted from "Dopamine signaling promotes the xenobiotic stress response and protein homeostasis," by K.K. Joshi, T. L. Matlack, and C. Rongo, 2016, The EMBO Journal, 35(17), 1885-1901 (doi: 10.15252/embj.201592524). Copyright 2016 by EMBO Press. Adapted with permission.

Cytochrome P450s (CYPs) are reduction-oxidation (redox) enzymes at the end of the electron transfer chain.' Loss-of-function mutants for various CYPs have previously been shown to stabilize polyubiquitinated substrates, suggesting decreased UPS activity. ${ }^{2}$ Certain CYPs also metabolize polyunsaturated fatty acids (PUFAs) to eicosanoid signalling molecules (Figure 2). PUFAs relevant to eicosanoid signalling can be divided into $\omega-3$ PUFAs, which have their first double bond at the third carbon, and $\omega-6$ PUFAs, which have their first double bond at carbon 6 .

\footnotetext{
1 Lamb et al., 2009

2 Joshi, Matlack, \& Rongo, 2016
} 


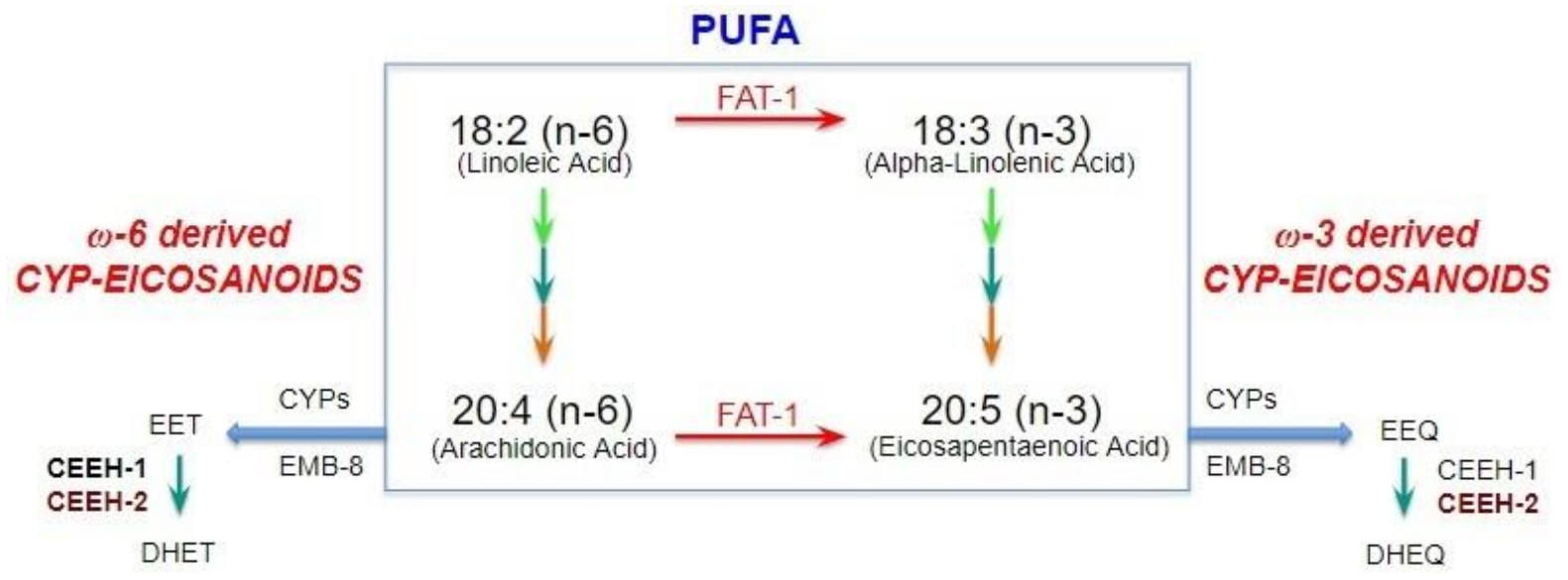

Figure 2. Schematic representation of $\omega-6$ and $\omega-3$ PUFAs and the CYP-eicosanoid derivative signaling pathway. $\omega-6$ PUFAs are converted to $\omega-3$ PUFAs via FAT-1 and PUFA-derived eicosanoids are produced via CYPs and EMB-8.

Thus, the eicosanoid synthesis pathway is a worthwhile target for study of UPS regulation. Here, various aspects of the eicosanoid synthesis pathway are studied in order to shed light on UPS regulation.

\section{Methods}

UPS activity can be monitored in Caenorhabditis elegans, a roundworm species, via a transgenic approach using the ubiquitin fusion degradation reporter transgene UbG76VGFP, which is an unstable protein covalently bonded to a green fluorescent protein (GFP). It mimics a monoubiquitinated protein that can be further polyubiquitinated and degraded. Once degraded, GFP is no longer detected (Figure 1). Therefore, GFP fluorescence in animals expressing UbG76V-GFP is inversely proportional to the level of UPS activity. The UbG76VGFP is expressed in epithelial cells from tissue-specific promoters-col- 19 in the hypodermis and sur-5 in the intestine. Two red fluorescent proteins, mRFP in the hypodermis and mCherry in the intestine, may be monitored alongside UbG76V-GFP and used as an internal standard for gene expression. Neither mRFP nor mCherry is ubiquitinated, so they do not get degraded. But, they are both expressed from the same promoter as GFP, allowing the quantification of gene expression by analyzing the ratios of GFP/mRFP and GFP/mCherry (Figure 1).

Essential fatty acids, specifically the $\omega-3$ PUFA alpha-linolenic acid (ALA), are important eicosanoid precursors that cannot be synthesized in the human body from monounsaturated fatty acids (MUFAs). However, $C$. elegans is able to convert other $\omega-6$ PUFAs to $\omega-3$ PUFAs using the FAT-1 gene, making it a model organism for eicosanoid study (Figure 2). Mutants of FAT-1 fail to make $\omega-3$ PUFAs and subsequently accumulate $\omega-$ 6 PUFAs. Studying these mutants can help determine whether either type of PUFA distinctly contributes to regulating the UPS. 
First, the odls77transgene expressing the UbG76V-GFP and mRFP reporters in the hypodermis was introduced into a fat-1(wa9) mutant strain and a control odls77 (wild-type) strain (Table 1). C. elegans undergoes four larval stages starting from L1 and ending at L4, the final 24-hour larval stage before the worm enters adulthood. A wild-type worm expressing UbG76V-GFP and mRFP in the hypodermis quickly accrues both proteins between the $L 4$ larval stage and the $L 4+24$ adult stage. Upon entering the $L 4+48$ adult stage, which is associated with peak fertility, UbG76V-GFP is swiftly degraded while mRFP remains relatively stable (Figure 1 ). Both the wild-type and fat- 1 strains were imaged at the L4+24 and L4+48 stages in order to determine UbG76V-GFP and mRFP levels through fluorescence analysis (Table 1).

To establish if UbG76V_GFP turnover with a fat- 1 mutant is as quick in the intestine as it is in the hypodermis, a fat-1(wa9); psur5 mutant strain homozygous for UbG76V_GFP was generated (Table 1). UbG76V_GFP expression in the intestine is less stable than expression in the hypodermis, suggesting earlier UPS activity in the intestine. This is possibly due to tissue-specific differences in proteasome demand, activity, or expression. Thus, there was a possibility of mistaking typical unstable UbG76V-GFP levels for a heterozygous genotype. Furthermore, in the intestine, UbG76V-GFP is on chromosome III; the RFP control, mCherry, is on chromosome $\mathrm{V}$; and the fat- 1 mutation is located on chromosome $\mathrm{V}$. So, there was a possibility of losing either fluorescent tag. Generating the intestinal fat- 1 strain in the lab allowed greater control over ensuring fluorescence. A pp608 (wild-type) strain, also homozygous for UbG76V-GFP and expressing UbG76V-GFP (chromosome III) and mCherry (chromosome V) from the sur-5 promoter, was used as a control to determine the impact of a fat- 1 mutation in the intestine on UPS activity. Both strains were imaged and analyzed at the $L 4+24$ and $L 4+48$ life stages (Table 1 ).

In C. elegans, proper CYP function requires the EMB-8 gene, which codes for a reductase enzyme that catalyzes electron transfer from NADPH to the CYP. ${ }^{3}$ CYPs require EMB- 8 to metabolize eicosanoids from PUFAs, so $e m b-8$ mutants fail to produce PUFA-derived CYPeicosanoids (Figure 2). Studying emb-8 mutants will help characterize the role of EMB- 8 eicosanoid derivatives in UPS regulation.

Because eicosanoids are essential signalling molecules in C. elegans, the emb- 8 null mutation is a lethal one. As a result, it is difficult to analyze the function of this gene in adult animals via a knockout study. To get around this issue, a temperature sensitive allele of $e m b-8$ called $e m b-8$ (hc69) was synthesized to determine how an $e m b-8$ mutation in the hypodermis impacts UPS activity. Specifically, the emb-8(hc69); odls 76 mutant strain and the control odls76 (wild-type) strain were generated and examined, both with the UbG76VGFP and mRFP reporter transgenes again expressed in the hypodermis (Table 1). Both strains were imaged and analyzed for fluorescence at the $L 4+24$ and $L 4+48$ stages. The L $4+72$ stage was also imaged for emb- 8 mutants to determine the extent of the stability of UbG76V-GFP (Table 1).

3 Benenati, Penkov, Muller-Reichert, Entchev, \& Kurzchalia, 2009 


\begin{tabular}{|c|c|c|c|c|c|c|c|c|}
\hline $\begin{array}{l}\text { Cell } \\
\text { Tissue }\end{array}$ & $\begin{array}{l}\text { Gene } \\
\text { Observed }\end{array}$ & $\begin{array}{l}\text { Control } \\
\text { Strain }\end{array}$ & Mutant Strain & $\begin{array}{l}\text { GFP } \\
\text { Standard }\end{array}$ & $\begin{array}{l}\text { RFP } \\
\text { Standard }\end{array}$ & $\begin{array}{l}\text { L4+24 } \\
\text { Imaged }\end{array}$ & $\begin{array}{l}\text { L4+48 } \\
\text { Imaged }\end{array}$ & $\begin{array}{l}\text { L4+72 } \\
\text { Imaged }\end{array}$ \\
\hline Epithelial & FAT-1 & $\begin{array}{l}\text { odls77 } \\
\text { (wild-type) }\end{array}$ & fat-1(wa9) & $U^{G} b^{G 6 V}$-GFP & mRFP & Yes & Yes & No \\
\hline Intestinal & FAT-1 & $\begin{array}{l}\text { pp608 } \\
\text { (wild-type) }\end{array}$ & $\begin{array}{l}\text { fat-1(wa9); } \\
\text { psur5 }\end{array}$ & $U_{b^{G 76 V}}$-GFP & mCherry & Yes & Yes & No \\
\hline Epithelial & EMB-8 & $\begin{array}{l}\text { odls76 } \\
\text { (wild-type) }\end{array}$ & $\begin{array}{l}\text { emb-8(hc69); } \\
\text { od/s76 }\end{array}$ & $U^{G} b^{G 6 V}$-GFP & mRFP & Yes & Yes & Yes $^{*}$ \\
\hline
\end{tabular}

*Only for the $e m b-8$ mutant strain

Table 1. Experimental setup. UbG76V_GFP/mRFP ratios were measured in the hypodermis at L4+24 hrs and L4+48 hrs for the fat- 1 mutant and odls 77 wild-type strains. UbG76V$\mathrm{GFP} / \mathrm{mRFP}$ ratios were measured in the hypodermis at L4+24 hrs, L4+48 hrs, and L $4+72 \mathrm{hrs}$ for the emb-8 mutant and pp608 wild-type strains. Ub $676 \mathrm{~V}-\mathrm{GFP} / \mathrm{mCherry}$ ratios were measured in the intestine at L4+24 hrs and L4+48 hrs for the fat -7 mutant and odls 76 wild-type strains.

\section{Results}

Accelerated UbG76V-GFP turnover in fat-1 mutants in the hypodermis

As expected, we observed that the wild-type strain exhibited accumulated UbG76V-GFP at the L4+24 stage, with similar levels of both Ub $676 \mathrm{~V}$-GFP and mRFP, giving a mean normalized ratio close to 1 (Figures $3 \& 5$; Table 2 ). This is consistent with accelerated protein accumulation seen at the end of the final larval stage as a wild-type worm enters adulthood, likely due to reproduction-associated protein synthesis. ${ }^{4}$ The wild-type strain exhibited reduced UbG76V-GFP levels at the L4+48 stage compared to the internal control mRFP, with a mean $\mathrm{UbG} \mathrm{G} \mathrm{V}_{-} \mathrm{GFP} / \mathrm{mRFP}$ ratio closer to 0 (Figures $7 \& 9$; Table 2 ). This is expected in the hypodermis of wild-type worms that have reached peak fertility, possibly as a protective mechanism against damage caused by protein aggregation. 5 The wild-type strain expressed mRFP to the same intensity at both stages, which indicates that non-ubiquitinated proteins in the hypodermis of wild-type worms are stable (Figures 1, 5, \& 9). By contrast, the fat- 1 mutant strain showed increased UbG76V_GFP turnover, showing reduced UbG76V_GFP levels and a mean $\mathrm{Ub}^{\mathrm{C} 76 \mathrm{~V}} \mathrm{G} \mathrm{GFP} / \mathrm{mRFP}$ ratio close to 0 at both stages (Figures $4 \& 8$; Table 2). Again, in this strain, mRFP remained stable at both stages, which is standard of non-ubiquitinated proteins (Figures $6 \& 10$ ).

\footnotetext{
4 Joshi et al., 2016

5 Joshi et al., 2016
} 
Aresty Rutgers Undergraduate Research Journal, vol. 1, issue 1, Spring 2020
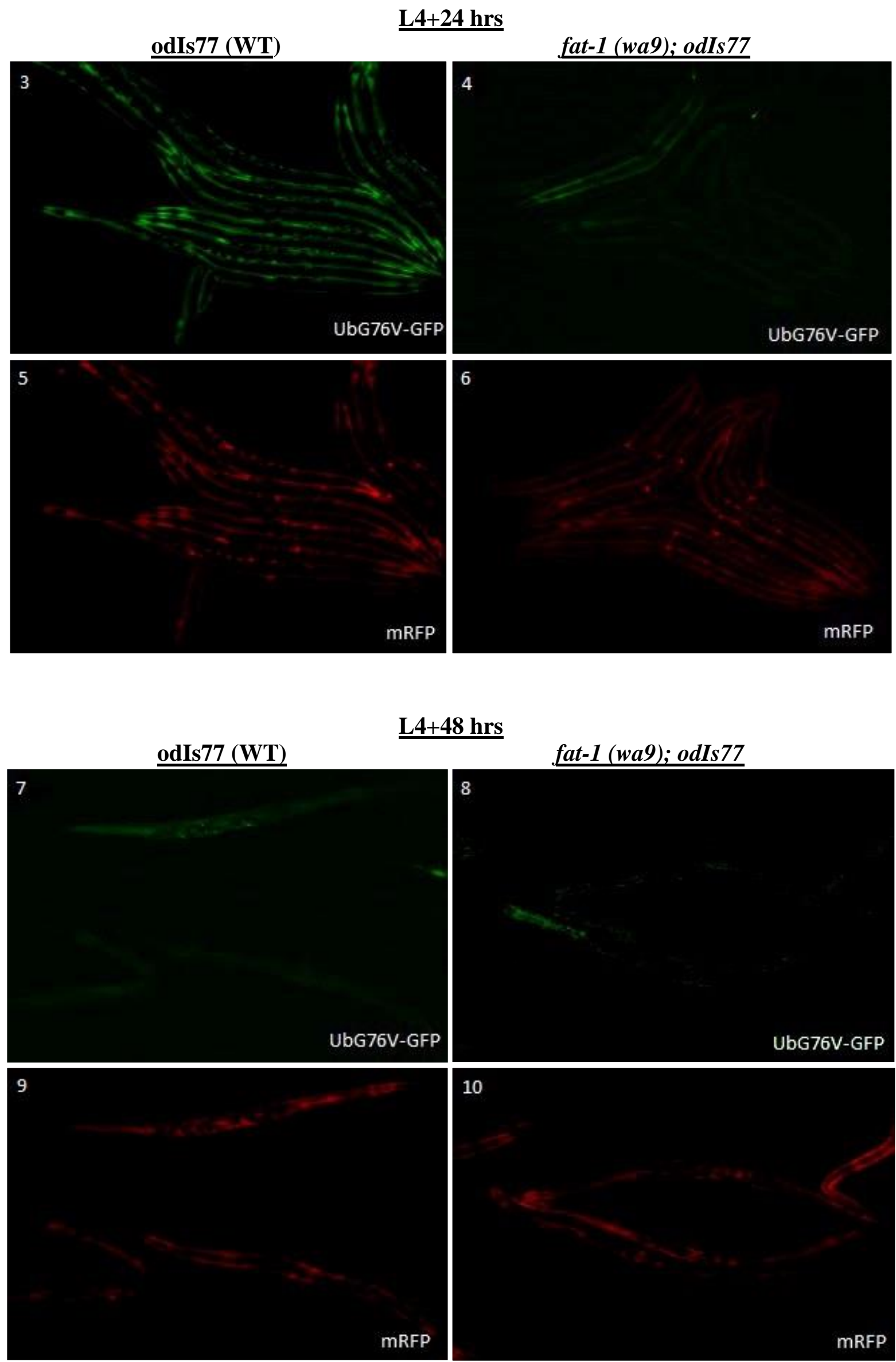
Figures 3-10. UbG76V-GFP turnover is accelerated in the hypodermis of fat- 1 mutants. 3 Expression of UbG76V-GFP (green) and 5 mRFP (red) from the col- 19 promoter at L4+24 hrs in wild-type animals. 4 Expression of UbG76V-GFP (green) and 6 mRFP (red) from the col- 19 promoter at L4 +24 hrs in fat -1 mutants. 7 Expression of UbG76V-GFP (green) and 9 mRFP (red) from the col- 19 promoter at $\mathrm{L} 4+48 \mathrm{hrs}$ in wild-type animals. 8 Expression of UbG76V_GFP (green) and $10 \mathrm{mRFP}$ (red) from the col-19 promoter at $L 4+48$ hrs in fat -1 mutants.

\begin{tabular}{|l|l|l|}
\hline & $\begin{array}{l}\text { Mean Ub } \\
\text { L4+24 hours }\end{array}$ & $\begin{array}{l}\text { Mean Uf } \text { Ub }^{G 76 V} \text {-GFP/mRFP ratio at } \\
\text { L4+48 hours }\end{array}$ \\
\hline Wild-Type & 1.066 & 0.02700 \\
\hline Fat-1 mutant & 0.05553 & 0.04469 \\
\hline
\end{tabular}

Table 2. fat- 1 mutants show accelerated UbG76V-GFP turnover in the hypodermis at $L 4+24$ hrs. Mean UbG76V_GFP/mRFP ratios at L4+24 hrs and L4+48 hrs in the hypodermis of fat $-\mathrm{l}$ mutant and wild-type strains.

At the $L 4+24$ stage, the variation in UbG76V-GFP expression between the wild-type strain and the fat- 1 mutant strain was significant $(p<0.0001)$ (Figure 11$)$. UbG76V-GFP levels in the wild-type strain were noticeably higher than in the fat- 7 mutant strain at this stage. At the L4+48 stage, both strains expressed low UbG76V_GFP levels $(p<0.01)$ (Figure 12). Taken together, these results suggest that the UPS is activated earlier the hypodermis of fat- 1 mutants than in the wild-type strain. As discussed, fat- 1 mutants are unable to convert $\omega-6$ PUFAs to $\omega-3$ PUFAs, which leads to an accumulation of the former and a corresponding deficit in the latter (Figure 2). Therefore, there are two mechanisms by which a fat-1 mutation can cause earlier UPS activation. First, $\omega-6$ PUFAs or their eicosanoid derivatives could promote the UPS, so their accumulation would cause premature UPS activity. Alternatively, $\omega-3$ PUFAs or their eicosanoid derivatives could inhibit the UPS, so their deficiency would accelerate UPS activity.

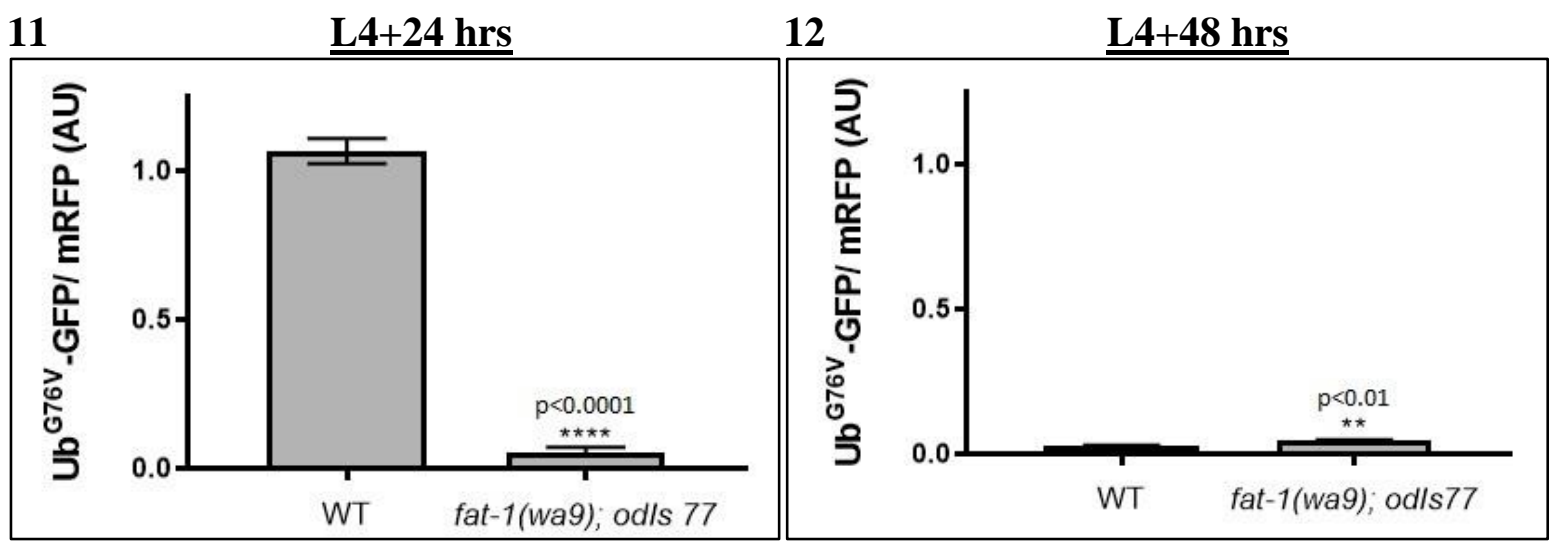

Figures 11 and 12. Graphical representation of accelerated UbG76V-GFP turnover in the hypodermis of fat-1 mutants. 11 Quantified fluorescence ratios of UbG76V-GFP to mRFP in the hypodermis of 20 
animals of specified strains at L4+24 hrs. ${ }^{*} * * * 0<00.0001$. Error bars denote SEM. 12 Quantified fluorescence ratios of UbG76V-GFP to mRFP in the hypodermis of 20 animals of specified strains at L4+48 hrs. $* * p<0.01$. Error bars denote SEM.

\section{Reduced UbG76V-GFP turnover in fat-1 mutants in the intestine}

As predicted in the intestine, the wild-type strain exhibited a mean UbG76V-GFP/mCherry ratio close to 0 at both the $L 4+24$ and L4+48 stages (Table 3); UbG76V_GFP fluorescence and expression was low in wild-type worms at both stages (Figures $13 \& 17$ ). This is consistent with the intestinal Ub ${ }^{G 76 V}$-GFP instability and potentially high underlying UPS activity observed in wild-type worms in prior studies. ${ }^{6}$ As the internal control, mCherry remained stable at both stages (Figures 15 \& 19). In the fat- 1 strain, UbG76V_GFP levels in the intestine remained stable (and clearly higher than the wild-type strain) at both the L4+24 and L4+48 stages with slightly reduced levels at the latter stage (Figures $14 \& 18$ ). Once again, the mCherry standard remained stable at both stages (Figures $16 \& 20$ ). Thus, the fat- 1 mutant strain displayed a UbG76V_GFP/mCherry ratio close to 1 at the L4+24 stage. This UbG76V $\mathrm{GFP} / \mathrm{mCherry}$ ratio was only moderately reduced at the L4+48 stage, further suggesting increased UbG76V-GFP stability in the intestine of fat- 1 mutants (Table 3). Given previous results with the fat- $l$ mutant in the hypodermis, it is possible that UPS activity and its regulation are subject to tissue-specific differences.

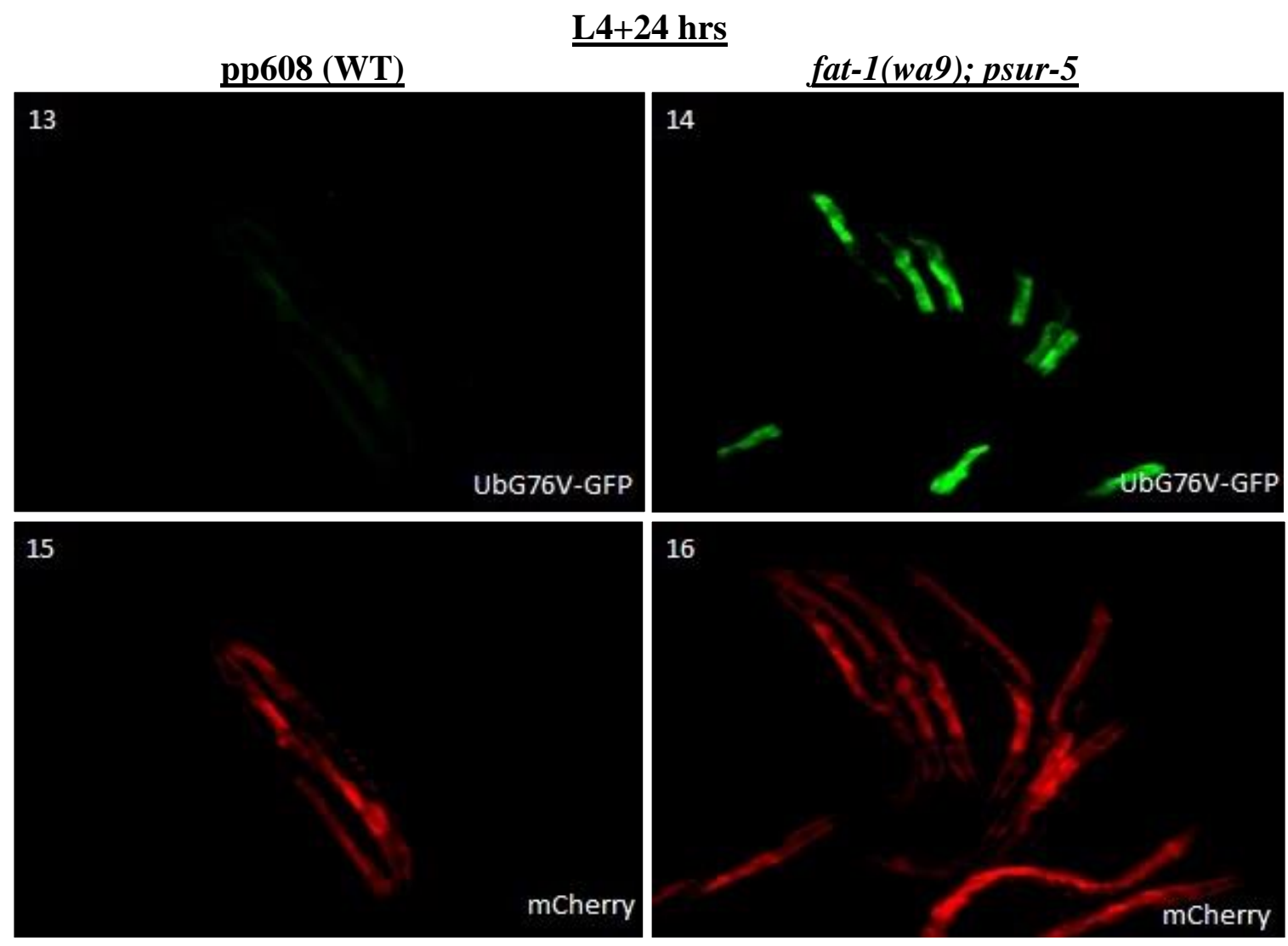

6 Keith et al., 2016 


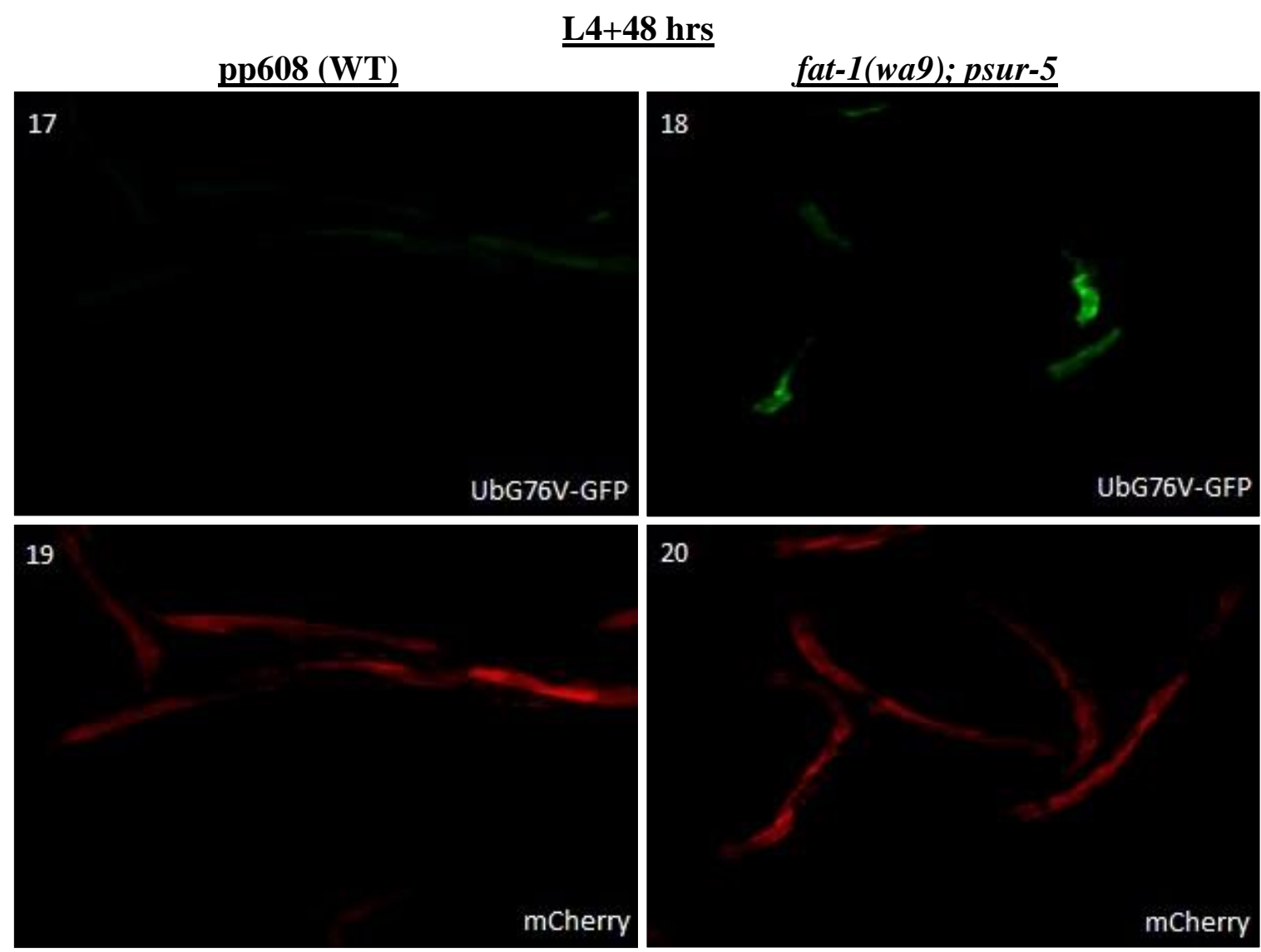

Figures 13-20. UbG76V-GFP turnover is reduced in the intestine of fat-1 mutants. 13 Expression of UbG76V-GFP (green) and 15 mCherry (red) from the sur-5 promoter at L4+24 hrs in wild-type animals. 14 Expression of UbG76V-GFP (green) and 16 mCherry (red) from the sur-5 promoter at L4+24 hrs in fat -1 mutants. 17 Expression of UbG76V-GFP (green) and 19 mCherry (red) from the sur-5 promoter at L4 +48 hrs in wild-type animals. 18 Expression of UbG76V-GFP (green) and 20 mCherry (red) from the sur -5 promoter at $L 4+48$ hrs in fat- 1 mutants.

\begin{tabular}{|l|l|l|}
\hline & $\begin{array}{l}\text { Mean Ub } \\
\text { L4+24 hours }\end{array}$ & $\begin{array}{l}\text { Mean UbP/mCherry ratio at6V_GFP/mCherry ratio at } \\
\text { L4+48 hours }\end{array}$ \\
\hline Wild-Type & 0.1016 & 0.09063 \\
\hline Fat-1 mutant & 0.9680 & 0.4275 \\
\hline
\end{tabular}

Table 3. fat- 1 mutants show reduced UbG76V_GFP turnover in the intestine at L4+48 hrs. Mean UbG76V $\mathrm{GFP} / \mathrm{mCherry}$ ratios at $\mathrm{L} 4+24 \mathrm{hrs}$ and $\mathrm{L} 4+48 \mathrm{hrs}$ in the intestine of fat -1 mutant and wild-type strains.

At both stages, the variation in the UbG76V-GFP fluorescence and expression between the two strains was significant $(p<0.0001)$ (Figures $21 \& 22)$. UbG76V_GFP levels in the intestine of the wild-type strain were lower compared to the fat- 1 mutant strain at both stages. These results suggest that, in the intestine of fat- 7 mutants, UPS activity is decreased compared to 
wild-type animals. This contrasts with the hypodermis of fat- 7 mutants, which exhibits increased UPS activity relative to the wild-type strain. Therefore, PUFAs (or their eicosanoid derivatives) in the intestine may have pathways inverse to those in the hypodermis. These results are consistent with a mechanism in which either the $\omega-6$ PUFA line inhibits UPS activity, or the $\omega-3$ PUFA line promotes UPS activity (Figure 2). Interestingly, wild-type worms also appear to differ in UPS activity in the hypodermis and the intestine, with the intestine experiencing earlier UPS activity than the hypodermis. ${ }^{7}$ In total, these results suggest that PUFAs or their eicosanoid derivatives regulate UPS activity in a tissue-specific fashion.

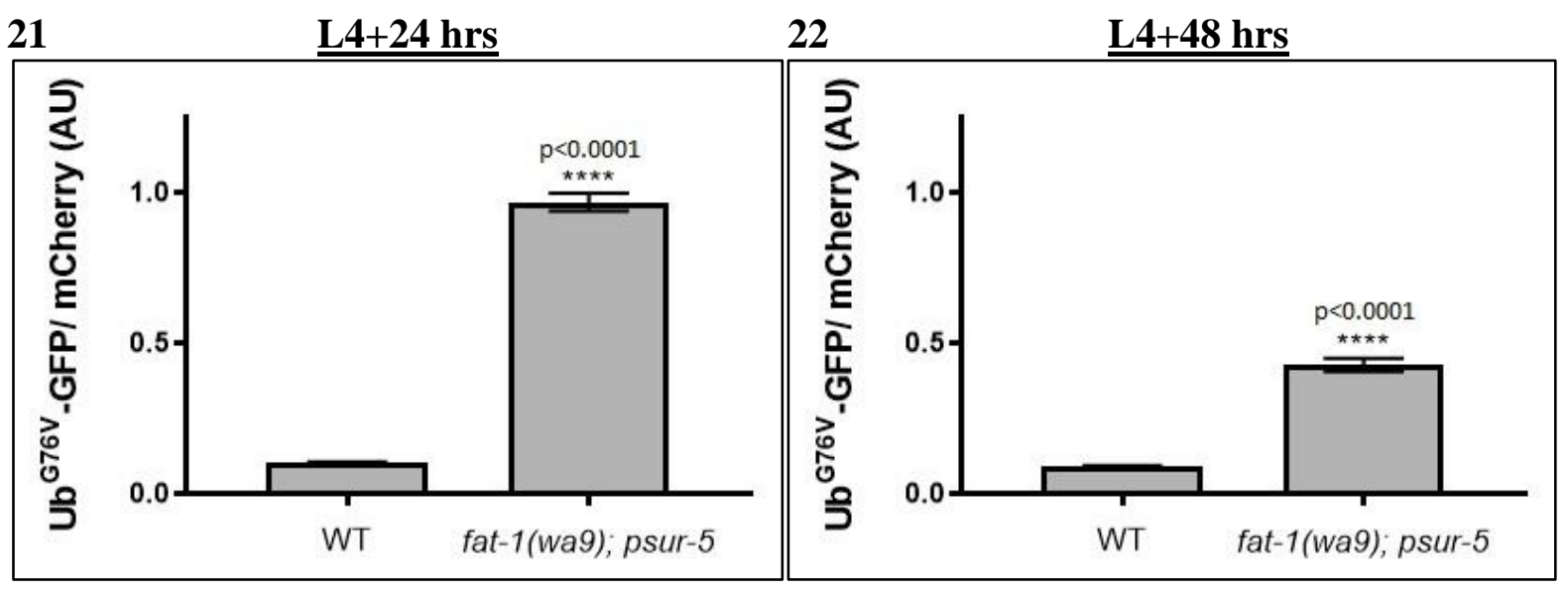

Figures 21 and 22. Graphical representation of reduced UbG76V_GFP turnover in the intestine of fat-1 mutants. 21 Quantified fluorescence ratios of UbG76V_GFP to mCherry in the intestine of 20 animals of specified strains at L4+24 hrs. ****p $<0.0001$. Error bars denote SEM. 22 Quantified fluorescence ratios of UbG76V-GFP to mCherry in the intestine of 20 animals of specified strains at L4+48 hrs. $* * * * p<0.0001$. Error bars denote SEM.

\section{Reduced UbG76V-GFP turnover in emb-8 mutants in the hypodermis}

In the hypodermis, wild-type $C$. elegans expressed UbG76V-GFP at elevated levels with a

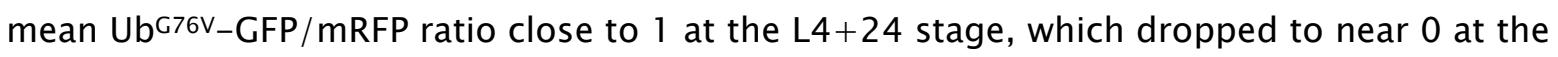
L4+48 stage (Figures $23 \& 27$; Table 4). As expected increased protein accumulation was seen in the hypodermis of wild-type worms when they first enter adulthood at L4+24 hours, and reduced protein levels were seen in the hypodermis of wild-type worms that reached peak fertility at L4+48 hours. ${ }^{8}$ The internal standard, mRFP, remained stable at both stages (Figures 25 \& 29). Similarly, emb-8 mutants also exhibited accumulated UbG76V_GFP levels at the $L 4+24$ stage with a mean UbG76V-GFP/mRFP ratio close to 1 . However, emb-8 mutants exhibited only slightly reduced UbG76V-GFP levels and UbG76V_GFP/mRFP ratios at the $L 4+48$ and L4+72 stages (Figures 24, 28, \& 31; Table 4). At both of the later stages, UbG76V_GFP levels were stable compared to the wild-type strain at the $L 4+48$ stage. Once again, as a control, mRFP remained stable in the emb-8 mutant strain at all three stages (Figures 26, $30, \& 32)$.

\footnotetext{
7 Keith et al., 2016

8 Joshi et al., 2016
} 
Aresty Rutgers Undergraduate Research Journal, vol. 1, issue 1, Spring 2020
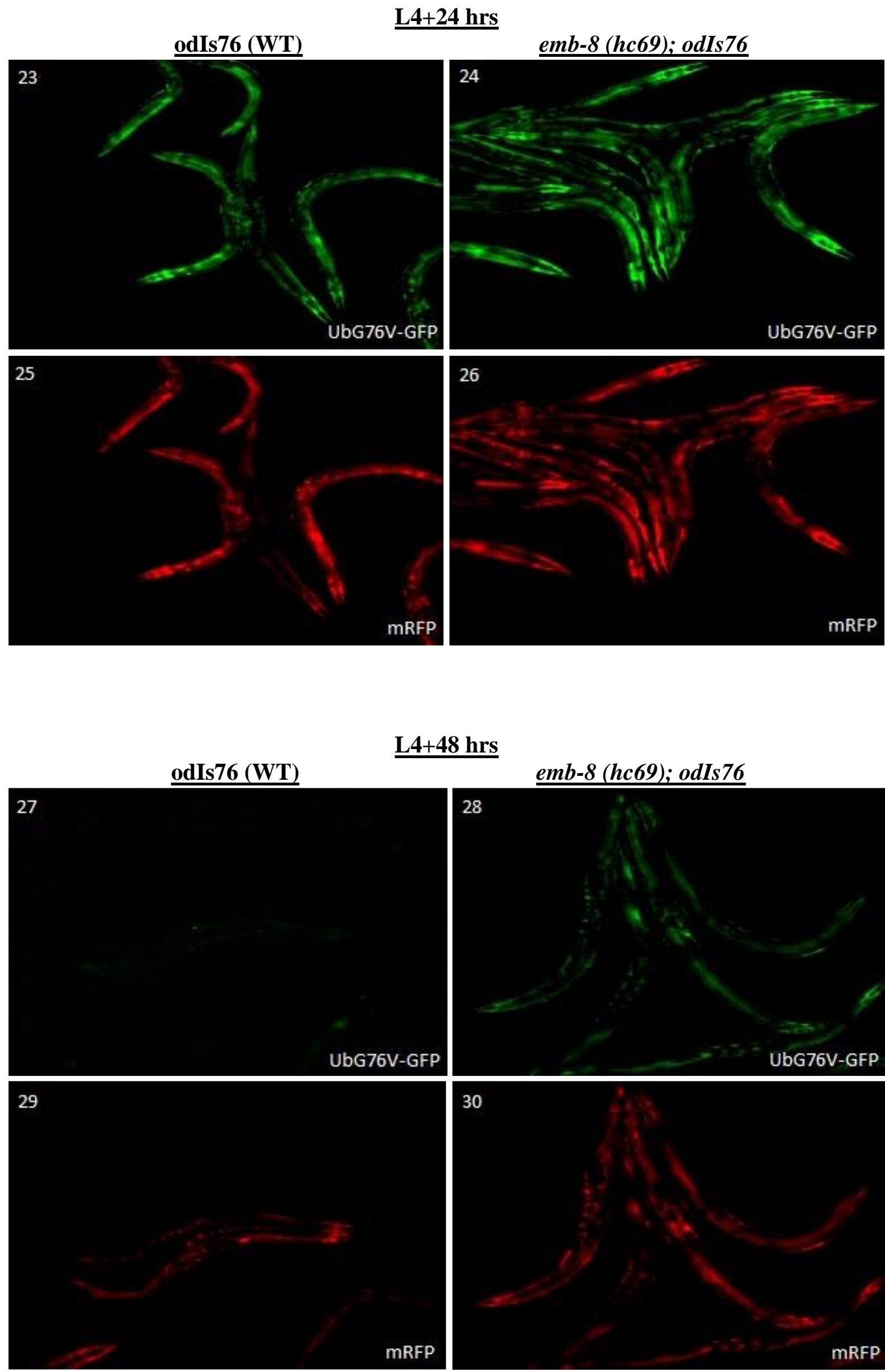


\section{L4+72 hrs: emb-8 (hc69); odIs76}
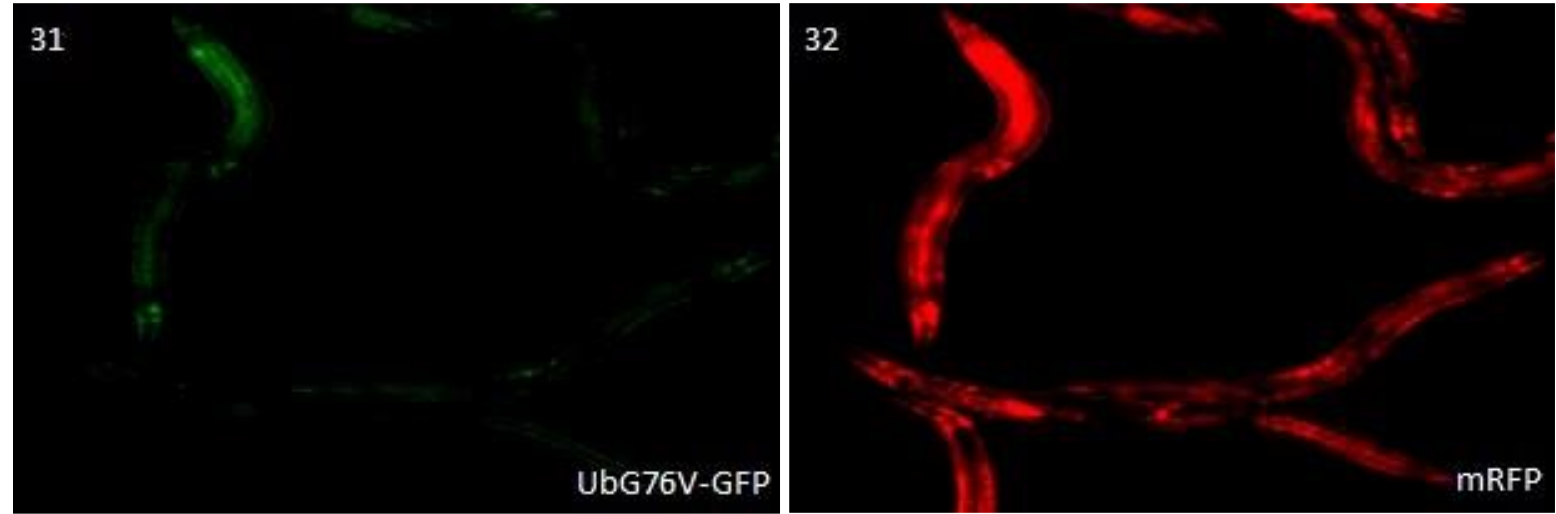

Figures 23-32. UbG76V-GFP turnover is reduced in the hypodermis of emb-8 mutants. 23 Expression of UbG76V_GFP (green) and 25 mRFP (red) from the col- 19 promoter at L4+24 hrs in wild-type animals. 24 Expression of UbG76V-GFP (green) and 26 mRFP (red) from the col- 19 promoter at L4+24 hrs in $e m b-8$ mutants. 27 Expression of UbG76V-GFP (green) and 29 mRFP (red) from the col- 19 promoter at L4+48 hrs in wild-type animals. 28 Expression of UbG76v-GFP (green) and $30 \mathrm{mRFP}$ (red) from the col19 promoter at L $4+48$ hrs in emb-8 mutants. 31 Expression of UbG76V-GFP (green) and 32 mRFP (red) from the col- 19 promoter at L $4+72 \mathrm{hrs}$ in emb- 8 mutants.

\begin{tabular}{|c|c|c|c|}
\hline & $\begin{array}{l}\text { Mean } \\
\text { Ub } \\
\text { ratio at } L 4+24 \text { hours }\end{array}$ & $\begin{array}{l}\text { Mean } \\
\text { Ub } \\
\text { ratio at } L 4+48 \text { hours }\end{array}$ & $\begin{array}{l}\text { Mean } \\
\text { Ub } \\
\text { ratio at } L 4+72 \text { hours }\end{array}$ \\
\hline Wild-type & 0.9317 & 0.07649 & N/A \\
\hline$E m b-8$ mutant & 0.9913 & 0.5931 & 0.2770 \\
\hline
\end{tabular}

Table 4. emb-8 mutants show reduced UbG76V-GFP turnover in the hypodermis at L4+24 hrs. Mean UbG76V-GFP/mRFP ratios at L4+24 hrs and L4+48 hrs in the hypodermis of emb- 8 mutant and wildtype strains. Mean UbG76V-GFP/mRFP ratio at L4+72 hrs is also shown for the emb- 8 mutant strain.

At the L4+24 stage, the variation in UbG76V_GFP fluorescence and expression between the wild-type and $e m b-8$ mutant strains was not significant $(p>0.05)$ (Figure 33). These results are unsurprising in the hypodermis, given that UbG76V_GFP accumulated at L4+24 hours in both strains. And, this aligns with increased protein levels and low UPS activity seen in the hypodermis of wild-type animals when they initially enter adulthood. ${ }^{9}$ However, at the L4+48 stage, the difference in UbG76V_GFP fluorescence and expression was highly significant $(p<0.0001)$ (Figure 34). Although UbG76V-GFP levels were reduced in the wildtype strain at $L 4+48$ hours, UbG76V-GFP levels in emb- 8 mutants remained stable at $\mathrm{L} 4+48$ hours. ${ }^{10}$ In the eicosanoid synthesis pathway, the EMB-8 gene is involved in the eventual conversion of both $\omega-6$ and $\omega-3$ PUFAs to their eicosanoid derivatives (Figure 2). Thus, if an $e m b-8$ mutant stabilizes UbG76V-GFP in the hypodermis by decreasing UPS activity, then

\footnotetext{
9 Joshi et al., 2016

10 Joshi et al., 2016
} 
these results support an eicosanoid-regulated pathway of the UPS, as opposed to a PUFAregulated pathway. It is likely that either $\omega-6$ PUFA-derived eicosanoids promote the UPS or $\omega-3$ PUFA-derived eicosanoids repress it. As a whole, these results indicate that UPS activity is suppressed in the hypodermis in emb-8 mutants.

33 L4+24 hrs 34 L4+48 hrs

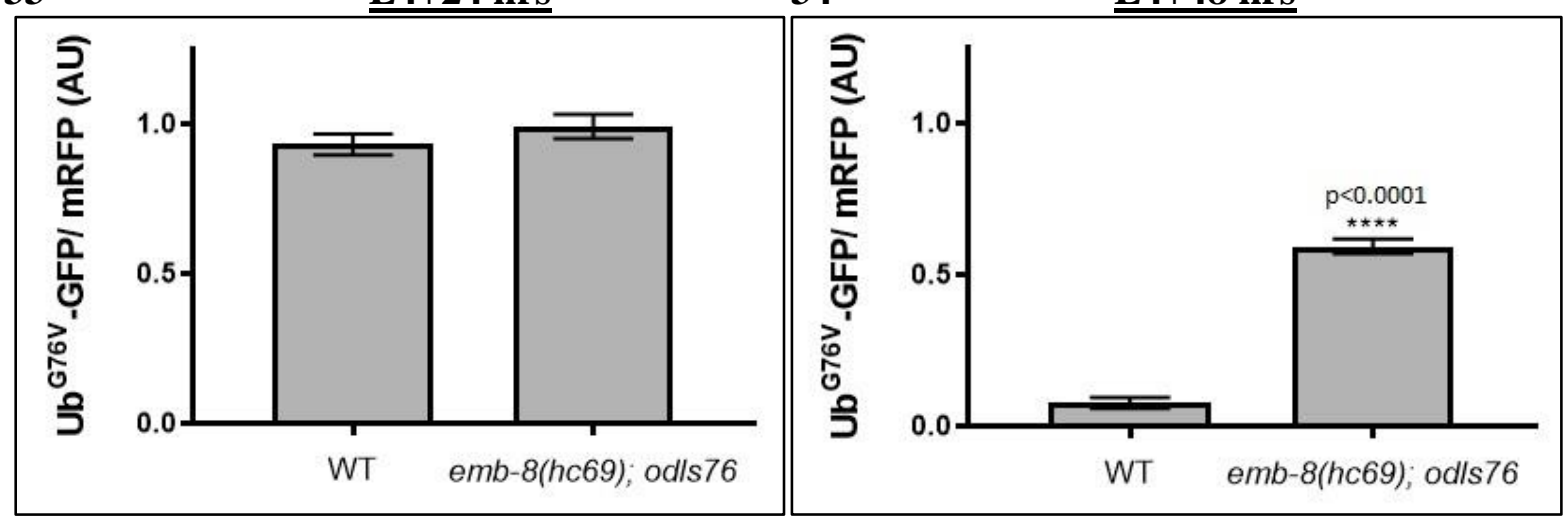

Figures 33 and 34. Graphical representation of reduced UbG76V_GFP turnover in the hypodermis of emb-8 mutants. 33 Quantified fluorescence ratios of UbG76V-GFP to mRFP in the hypodermis of 20 animals of specified strains at $L 4+24 \mathrm{hrs}$. Lack of a $p$-value indicates $p>0.05$. Error bars denote SEM. 34 Quantified fluorescence ratios of UbG76V-GFP to mRFP in the hypodermis of 20 animals of specified strains at $L 4+48$ hrs. $* * * * \mathrm{p}<0.0001$. Error bars denote SEM.

\section{Discussion}

In order to characterize the role of eicosanoids in the regulation of UPS activity and proteostasis, a UbG76V_GFP transgene was introduced into various $C$. elegans mutants to monitor changes in UPS activity, which is inversely related to UbG76V_GFP intensity. In wildtype $C$. elegans strains, UbG76V-GFP begins to accrue at the L4+24 stage and is swiftly degraded when worms enter peak fertility at the L4+48 stage. In this work, it was confirmed that several constituents of the fatty acid-eicosanoid signalling pathway, namely FAT- 1 and EMB-8, are involved in mediating the UPS. In the hypodermis, fat -1 mutants experienced earlier UPS activation relative to wild-type animals, while emb-8 mutants exhibited repressed UPS activity. In the intestine, however, fat- 7 mutants displayed UPS repression. These findings suggest that eicosanoids function in a tissue-specific manner to regulate the UPS and maintain proteostasis.

Two mechanisms within the fatty-acid-eicosanoid pathway can be proposed for the increased Ub ${ }^{676 \mathrm{~V}}$-GFP turnover observed in the hypodermis of a fat- 1 mutant. Mutants for FAT- 1 accumulate $\omega-6$ PUFAs because they cannot convert them to $\omega-3$ PUFAs. Therefore, one possible explanation is that $\omega-6$ derived CYP-eicosanoids promote UPS activity. This means a fat- 1 mutation leads to enhanced UPS activity in the hypodermis due to high levels of one or more $\omega-6$ PUFAs or their eicosanoid derivatives. Another potential explanation is that $\omega-3$ PUFAs or their eicosanoid derivatives inhibit UPS activity, so their absence due to 
fat- 7 results in the de-repression of hypodermal UPS activity. Since the results presented here do not suggest one pathway over the other, further studies are required to determine which one of these two pathways-if not a combination of both-is responsible for the effects of FAT-1 on UPS activity.

Curiously, fat- 7 in the intestine increases UPS activity, which is the opposite of its inhibitory role in the hypodermis. Thus, it is likely that eicosanoids exert their effects on the UPS in a cell and tissue-specific manner. This is supported by previous studies that found the effects of $\omega-3$ PUFAs varied depending on the tissue in question, with reduced UPS activity in cachexia cancer cells and increased UPS activity in HPV-infected cancer cells. ${ }^{11}$ In the intestine, $\omega-3$ PUFAs or their derived eicosanoids may promote UPS activity, or $\omega-6$ PUFAs or their derived eicosanoids may inhibit it. The exact purpose of tissue-specific differentiation in UPS regulation is unknown, although it is possible that there is a greater demand to break down proteins in the intestine than in the hypodermis.

Together with CYPs, EMB-8 is responsible for the direct production of eicosanoids. The stabilization of $\mathrm{Ub}^{\mathrm{G} 76 \mathrm{~V}}$-GFP resulting from an $e m b-8$ mutation in the hypodermis likely suggests a mechanism in which eicosanoids promote UPS activity. This is further supported by the observations in the hypodermis of a fat- 7 mutant. To determine whether it is $\omega-6$ PUFAs or $\omega-6$ derived eicosanoids regulating the UPS in the hypodermis, it would be worthwhile to study a fat- 1 and $e m b-8$ (or even a $c y p$ ) double mutant and observe whether the emb- 8 mutation blocks the increased UbG76V_GFP turnover induced by the fat- 1 mutation. The same experiment could be repeated in the intestine to determine the role of eicosanoids in regulating the UPS in a different type of cell. Double mutations in FAT- 1 and other FAT enzymes would also be valuable in determining the contribution of different $\omega-6$ PUFAs to UPS regulation and if the effect arises from their derived eicosanoids instead. Studying mutations in $\mathrm{CEEH}-1$ and $\mathrm{CEEH}-2$-which convert nonclassical, short-lived eicosanoids to classic eicosanoids-would also be helpful in the latter case (Figure 2).

Although the results in this paper have been discussed in the context of eicosanoids regulating the UPS, it is also possible that they function through regulating other protein degradation mechanisms, such as autophagy or even proteasome activity itself. One study observed that supplementing growth media with $\omega-6$ PUFAs activates autophagy in $C$. elegans, which serves to increase starvation resistance and elongate their lifespans. 12 Another publication found that $\omega-3$ PUFAs increased the concentration of ubiquitinated proteins in adipocytes, crediting it to reduced expression of proteasome subunits. ${ }^{13}$ Although previous studies have examined mutations in possible regulators of the UPS in conjunction with direct disruption of the proteasome, the exact role of eicosanoids in modulating proteostasis remains unclear. ${ }^{14}$

Regardless of their precise function, eicosanoids are key components in eukaryotic proteostasis, including protein degradation. Beyond C. elegans, understanding the role of

\footnotetext{
11 Whitehouse, Smith, Drake, \& Tisdale, 2001; Jing et al., 2014

12 O’Rourke, Kuballa, Xavier, \& Ruvkun, 2013

13 Wójcik et al., 2014

14 Joshi et al., 2016
} 
eicosanoids synthesized from essential fatty acids in proteostasis will help to elucidate their biological importance. Improving our understanding of the UPS system may help us to better comprehend and prevent age-associated neurodegeneration and disease.

\section{Materials and Procedures}

\section{Strains used}

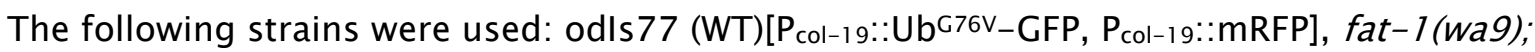

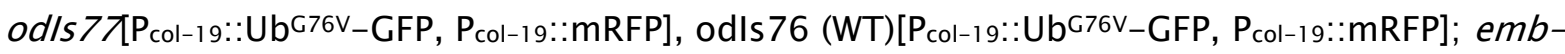

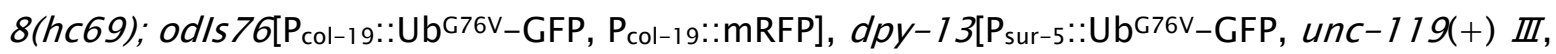

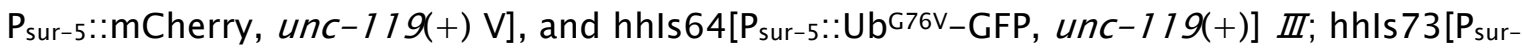
5::mCherry, unc- $119(+)] \mathrm{V}$.

\section{Growth conditions}

Under standard conditions, worms were grown at $20^{\circ} \mathrm{C}$ on nematode growth media (NGM) plates seeded with OP50 Escherichia coli. The temperature-sensitive emb-8(hc69); odls 76 strain was grown at $15^{\circ} \mathrm{C}$ until the post-L4 stage, when it was shifted to $20^{\circ} \mathrm{C}$, along with the wild-type control, odls76.

To generate the fat-1(wa9); psur-5 strain, the $d p y-13$; psur-5 strain was used as a balancer to introduce psur5 into the fat-1(wa9) strain. 15 hermaphrodites of the $d p y-13$; psur-5 strain were first crossed to 20 laboratory N2 (WT) males. Males of the heterozygous F 1 generation were crossed to fat-l(wa9) hermaphrodites. $20 \mathrm{~F} 2$ progeny were separated onto 20 plates and self-crossed. From F3 plates that produced both wild-type and Dpy worms, 40 phenotypically wild-type worms were separated onto 40 plates and self-crossed. 25 F4 progeny from $\mathrm{F} 3$ plates that produced only phenotypically wild-type progeny were separated onto 25 plates. All worms separated displayed UbG76V_GFP and mCherry fluorescence. To test if UbG76V_GFP could be observed in the fat-1(wa9); psur-5 strain, $5 \mathrm{~F} 5$ progeny from each of the 25 F4 plates were separated onto 25 ufd- 7 RNAi plates to knock down ubiquitination in the worms. Five worms for the odls77 (WT) strain were also separated onto a $u f d-7$ RNAi plate as a control. Worms from the pp608 (WT) control strain were confirmed to be homozygous for Ub $\mathrm{b}^{\mathrm{C}} \mathrm{V}$-GFP by growing on a single $u f d-7$ RNAi plate.

\section{Statistics}

A t-test with Welch's correction was used in GraphPad Prism to analyze UbG76V_GFP and mRFP or mCherry fluorescence values for twenty worms per strain per life stage. Each strain's values were then graphed in GraphPad Prism.

\section{Bleaching and synchronization}

Gravid adult worms were harvested with M9 buffer and lysed with modified bleaching solution prepared in distilled water containing $10 \% 10 \mathrm{~N} \mathrm{NaOH}$ and $10 \%$ commercially 
available bleaching solution. Eggs were resuspended in 20-70 $\mu \mathrm{l}$ of M9 buffer and spotted onto NGM plates seeded with E. coli.

\section{Fluorescence microscopy, imaging, and intensity analysis}

Twenty worms per strain per life stage were imaged using a $5 x$ objective to detect UbG76V GFP, mRFP, and mCherry fluorescence with an epifluorescence microscope and iVision software. The worms were first mounted and paralyzed on slides with $2 \%$ agarose and $10 \mathrm{mM}$ tetramisole. The exposure times were $50 \mathrm{~ms}$ for $\mathrm{L} 4+24 \mathrm{hrs}$ and $25 \mathrm{~ms}$ for $\mathrm{L} 4+48$ and L $4+72$ hrs. ImageJ was used to outline individual worms in the images and quantify fluorescence. Python software was used to calculate mean fluorescence.

\section{RNAi screen}

Five individuals from each strain to be screened, including the wild-type control, were put on NGM plates with $25 \mu \mathrm{g} / \mathrm{mL}$ carbenicillin and $0.2 \%$ lactose. The plates were seeded with $E$. coli that produced dsRNA to knock down expression of UFD- 1 and allow assessment of UbG76V_GFP homozygosity. A second assessment was conducted by first synchronizing worms from each strain through bleaching, including the wild-type control, and spotting the eggs onto the RNAi plates. The worms were grown at $20^{\circ} \mathrm{C}$ and assessed for UbG76V-GFP homozygosity at the $L 4+24$ stage.

\section{Acknowledgements}

I am grateful towards Dr. Christopher Rongo for overseeing and supervising this research project and for providing valuable suggestions and directions for the research question. I would also like to express my gratitude towards Dr. Kishore Joshi for his direct supervision of the experiments and for his aid in learning laboratory techniques and background information. I also thank Nanci Kane, Mehul Vora, and Nathaly Salazar for their help with laboratory equipment and procedures.

\section{References}

Benenati, G., Penkov, S., Müller-Reichert, T., Entchev, E. V., \& Kurzchalia, T. V. (2009).Two cytochrome P450s in Caenorhabditis elegans are essential for the organization of eggshell, correct execution of meiosis and the polarization of embryo. Mechanisms of Development, 126, 382-393. doi: 10.1016/j.mod.2009.02.001

Ciechanover, A., \& Kwon, Y. T. (2015). Degradation of misfolded proteins in neurodegenerative diseases: therapeutic targets and strategies. Experimental \& Molecular Medicine, 47, 1-16. doi:10.1038/emm.2014.117

Jing, K., Shin, S., Jeong, S., Kim, S., Song, K-S., Park, J-H., ... Lim, K. (2014). Docosahexaenoic acid induces the degradation of HPV E6/E7 oncoproteins by activating the ubiquitin-proteasome system. Cell Death and Disease, 5. doi: $10.1038 /$ cddis. 2014.477 
Joshi, K. K., Matlack, T. L., \& Rongo, C. (2016). Dopamine signaling promotes the xenobiotic stress response and protein homeostasis. The EMBO Journal, 35(17), 1885-1901. doi: 10.15252 /embj.201592524

Keith, S. A., Maddux, S. K., Zhong, Y., Chinchankar, M. N., Ferguson, A. A., Ghazi, A., \&b Fisher, A. L. (2016). Graded Proteasome Dysfunction in Caenorhabditis elegans Activates an Adaptive Response Involving the Conserved $S K N-1$ and $E L T-2$ Transcription Factors and the Autophagy-Lysosome Pathway. PLOS Genetics, 12(2). doi: 10.1371 /journal.pgen.1005823

Lamb, D. C., Lei, L., Warrilow, A. G. S., Lepesheva, G. I., Mullins, J. G. L., Waterman, M. R., \& Kelly, S. L. (2009). The First Virally Encoded Cytochrome P450. Journal of Virology, 83(16), 8266-8269. doi:10.1128/JVI.00289-09

Liu, G., Rogers, J., Murphy, C. T., \& Rongo, C. (2011). EGF signalling activates the ubiquitin proteasome system to modulate C. elegans lifespan. The EMBO Journal, 30(15), 2990-3003. doi: 10.1038/emboj.2011.195

Lockhart-Jamieson, K., Endo, T., Darwesh, A. M., Samokhvalov, V., \& Seubert, J. M. (2017). Cytochrome P450-derived eicosanoids and heart function. Pharmacology \& Therapeutics, 179, 47-83. https://doi.org/10.1016/j.pharmthera.2017.05.005

O'Rourke, E. J., Kuballa, P., Xavier, R., \& Ruvkun, G. (2013). w-6 Polyunsaturated fatty acids extend life span through the activation of autophagy. Genes \& Development, 27(4), 429-440. doi: 10.1101/gad.205294.112

Powers, E. T., Morimoto, R. I., Dillin, A., Kelly, J. W., \& Balch, W. E. Biological and Chemical Approaches to Diseases of Proteostasis Deficiency. Annual Review of Biochemistry, 78, 959-991. doi: 10.1146/annurev.biochem.052308.114844

Rappleye, C. A., Tagawa, A., Le Bot, N., Ahringer, J., \& Aroian, R. V. (2003). Involvement of fatty acid pathways and cortical interaction of the pronuclear complex in Caenorhabditis elegans embryonic polarity. BMC Developmental Biology, 3(8). doi: $10.1186 / 1471-213 X-3-8$

Whitehouse, A. S., Smith, H. J., Drake, J. L., \& Tisdale, M. J. (2001). Mechanism of Attenuation of Skeletal Muscle Protein Catabolism in Cancer Cachexia by Eicosapentaenoic Acid. Cancer Research, 61 (9), 3604-3609.

Wójcik, C., Lohe, K., Kuang, C., Xiao, Y., Jouni, Z., \& Poels, E. (2014). Modulation of adipocyte differentiation by omega-3 polyunsaturated fatty acids involves the ubiquitin-proteasome system. Journal of Cellular and Molecular Medicine, 18(4), 590-599. doi: $10.1111 / \mathrm{jcmm} .12194$ 


\section{Appendix A}

\section{Definitions of Terms}

ALA. $\omega-3$ PUFA alpha-linolenic acid. Essential fatty acid that cannot be synthesized in the human body. Important eicosanoid precursor.

CEEH-1 and CEEH-2. Catalyze the hydrolysis of short-lived nonclassic eicosanoids to eicosanoids. Coded by the ceeh- 1 and ceeh-2 genes, respectively.

C. elegans. Caenorhabditis elegans. Free-living nematodes. Model species for many experiments.

col-19. Promoter from which UbG76V-GFP and mRFP are expressed from in the hypodermis. CYP. Cytochrome P450. Oxidation-reduction enzyme at the end of the electron transport chain. Facilitates the conversion of PUFAs to eicosanoids.

DHET and DHEQ. Dihydroxyeicosatrienoic acid and Dihydroxyeicosatrienoic acid. Derived from $\omega-6$ PUFAs and $\omega-3$ PUFAs, respectively. Eicosanoid signalling molecules. (Fig 1)

EET and EEQ. Epoxyeicosatrienoic acid and Epoxyeicosaquatraenoic acid. Derived from $\omega-6$ PUFAs and $\omega-3$ PUFAs, respectively. Short-lived nonclassic eicosanoid signalling molecules converted to eicosanoids by $\mathrm{CEEH}-1$ and $\mathrm{CEEH}-2$. (Fig 1)

EMB-8. Reductase enzyme required by CYPs to convert PUFAs to eicosanoids. Coded by the emb-8 gene.

$e m b-8$ (hc69); odls76. emb-8 mutant strain used in the hypodermis. Contains a temperature-sensitive allele. Typically a lethal mutation.

FAT-1. Desaturase enzyme that converts $\omega-6$ PUFAs to $\omega-3$ PUFAs. Coded by the fat- 1 gene.

fat-1 (wa9). fat-l mutant strain used in the hypodermis.

fat-1(wa9); psur5. fat-1 mutant strain used in the intestine.

GFP. Green fluorescent protein. Used as a reporter so that molecules can be imaged and analyzed.

UbG76V-GFP. Ubiquitin protein, composed of 76 amino acids, with the first being glycine and the final being valine, covalently attached to a GFP to construct an unstable transgene that can be introduced into research specimens.

L4 stage. Final larval stage of nematodes.

L4+24 stage. First adult stage of nematodes. 24 hours since nematode entered L4.

L4+48 stage. 48 hours since nematode entered L4.

L4+72 stage. 72 hours since nematode entered L4.

MUFA. Monounsaturated fatty acid. Fatty acids containing one double bond. Precursors to PUFAs.

odls76 (WT). Wild-type strain used as the control for the emb-8 mutant in the hypodermis. odls77 (WT). Wild-type strain used as the control for the fat- 1 mutant in the hypodermis. pp608 (WT). Wild-type strain used as the control for the fat- 1 mutant in the intestine. PUFA. Polyunsaturated fatty acid. Fatty acids containing more than one double bond. Precursors to eicosanoids.

$\omega-3$ PUFA. Contains first double bond at carbon 3. Converted from $\omega-6$ PUFAs by the FAT-1 desaturase enzyme.

$\omega-6$ PUFA. Contains first double bond at carbon 6 . Converted to $\omega-3$ PUFAs by the 
FAT-1 desaturase enzyme.

RFP. Red fluorescent protein. Used as a control reporter so that molecules can be imaged and analyzed.

mCherry. RFP reporter used in the intestine.

mRFP. RFP reporter used in the hypodermis.

sur-5. Promoter from which UbG76V_GFP and mCherry are expressed from in the intestine. UPS. Ubiquitin proteasome system. Mechanism through which damaged or unfolded proteins targeted for degradation by the proteasome via ubiquitin tagging. 